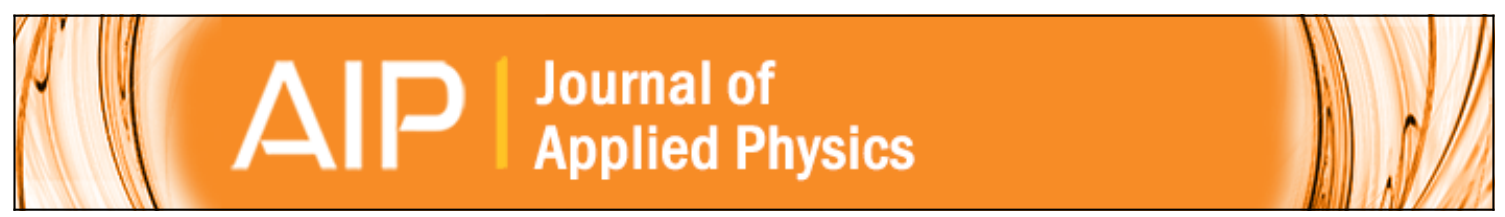

Variation of the refractive index and polarizability of sapphire under high pressures

N. M. Balzaretti, J. P. Denis, and J. A. H. da Jornada

Citation: Journal of Applied Physics 73, 1426 (1993); doi: 10.1063/1.353240

View online: $\mathrm{http}: / / d x . d o i . o r g / 10.1063 / 1.353240$

View Table of Contents: http://scitation.aip.org/content/aip/journal/jap/73/3?ver=pdfcov

Published by the AIP Publishing

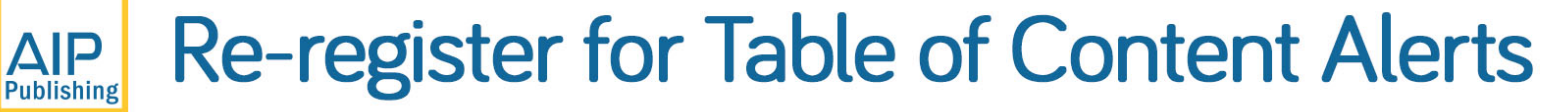




\title{
Variation of the refractive index and polarizability of sapphire under high pressures
}

\author{
N. M. Balzaretti \\ Instituto de Fisica, UFRGS, Porto Alegre, 91500 RS, Brazil \\ J. P. Denis \\ Laboratoire de Physico-Chimie des Materiaux, CNRS, Meudon 92190, France \\ J. A. H. da Jornada \\ Instituto de Fisica, UFRGS, Porto Alegre, 91500 RS, Brazil
}

(Received 18 May 1992; accepted for publication 9 October 1992)

\begin{abstract}
In this article we present the experimental results for the pressure variation of the refractive index of sapphire up to $16 \mathrm{GPa}$, obtained with an interferometric method, using the diamond anvil cell. In the range of hydrostatic pressures, up to about $12 \mathrm{GPa}$, the analysis of the results with the classic Lorentz-Lorenz approach provides a nearly linear relation between polarizability $(\alpha)$ and volume, corresponding to a constant strain polarizability parameter. For pressures above $12 \mathrm{GPa}$, there is a substantial nonlinear deviation, associated with nonhydrostatic effects inside the diamond anvil cell.
\end{abstract}

\section{INTRODUCTION}

The direct measurement of the variation of the refractive index with pressure is of practical importance in the design of modern optical systems for predicting the effects of stress or strain on the performance of optical components, especially for high-power laser applications. ${ }^{1}$ On the other hand, the photoelastic behavior is also an interesting subject from the theoretical point of view, related to microscopic properties of the crystal, such as the dependence of electronic polarizabilities with interionic distances.

In the literature, experimental results about the photoelastic behavior of materials over an extended region of pressure are scarce ${ }^{2}$ due to the inherent difficulties of the technique involved. The existing experimental works are mostly in the range up to $1.4 \mathrm{GPa}^{3,4}$ where there is usually a linear dependence between refractive index and volume. Waxler and Weir, ${ }^{5}$ in an early work, predicted qualitatively that a nonlinear behavior would be expected for higher pressures due to the changes of the interatomic forces.

Sapphire $\left(\mathrm{Al}_{2} \mathrm{O}_{3}\right)$ is a well studied and very important material used in optical windows. ${ }^{1}$ Moreover, the physical situation for the change of the refractive index under high pressures is relatively simple since the polarizability of the $\mathrm{Al}^{+3}$ ion is negligible compared to the $\mathrm{O}^{-2}$ ion, and the structure of this crystal, despite being hexagonal, behaves isotropically under hydrostatic compression, with the $c / a$ ratio remaining practically constant up to $12 \mathrm{GPa} .{ }^{6}$ Davis and Vedam, ${ }^{4}$ using an interferometric method, observed a linear decrease of both ordinary and extraordinary refractive indexes of sapphire up to $0.7 \mathrm{GPa}$.

In this article we present the results for a single crystal of sapphire, using an improved interferometric method ${ }^{7}$ over an extended region of hydrostatic pressures, up to 12 $\mathrm{GPa}$, where there is a considerable volume change of about $4.5 \%$, and nonlinear effects may appear.

\section{EXPERIMENTAL TECHNIQUE}

The experimental technique basically consists of following the transmitted interference peaks of a Fabry-Perot microinterferometer subjected to high hydrostatic pressures in a gasketed diamond anvil cell (DAC). This interferometer is a thin plate of sapphire, manually polished down to a thickness of about $40 \mu \mathrm{m}$, with the parallel faces covered by a semireflecting metallic film. These surfaces must be extremely flat and parallel in order to provide the maximum fringe sharpness. The design of the optical system used to obtain the interference spectrum of the microinterferometer inside the DAC is described in detail in a previous work. ${ }^{7}$ The pressure was determined by a ruby calibrant and the pressure-transmitting medium utilized was the 16:3:1 mixture of methanol, ethanol, and water.

The wavelength shift of the interference peaks is due to the change of the optical path of the light traversing the microinterferometer and, from the condition of maximum of interference for normal incidence, we can write ${ }^{7}$

$$
\Delta \lambda_{m}(P)=\lambda_{m}(0)\left(\frac{n(P)}{n_{0}} \frac{l(P)}{l_{0}}-1\right),
$$

where $\Delta \lambda_{m}(P)=\lambda_{m}(P)-\lambda_{m}(0)$ is the pressure shift of the peak of order $m, l$ is the thickness, and $n$ is the refractive index of the sample. The subscript " 0 " indicates $P=0$. According to this relation, the known pressure variation of the sample thickness can be used to get experimental results about the refractive index pressure dependence by following a given interference peak. Figure 1 shows typical channeled spectra for two different values of pressure, where the very good finesse and peak to background ratio can be seen, which enable a good accuracy in measuring changes of $n$ with $P$.

The spectral range covered by the selected fringe was from approximately $680 \mathrm{~nm}\left(n_{0}=1.736^{8}\right)$ to $650 \mathrm{~nm}$, where all the fringes were uniformly spaced and have practically the same intensity. We just corrected for the small 


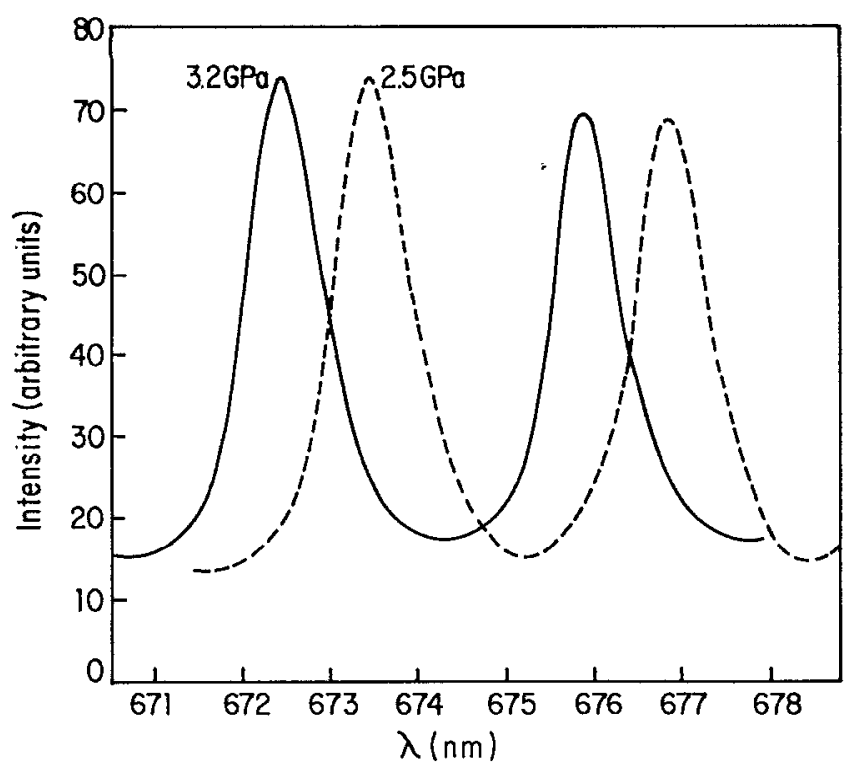

FIG. 1. Typical interference spectra for $P=3.2 \mathrm{GPa}(-)$ and $P=2.5$ $\mathrm{GPa}(--)$, showing the respective wavelength shift.

effect of dispersion of the refractive index by using the following approximation

$$
n(P, \lambda)=n\left(P, \lambda_{0}\right)+\left(\frac{\delta n}{\delta \lambda}\right)_{\lambda_{0}} \Delta \lambda,
$$

where $(\delta n / \delta \lambda)_{\lambda_{0}}=-3.8 \times 10^{-5} / \mathrm{nm}^{8}$

Sapphire is a hexagonal birefringent crystal, so we prepared the microinterferometer with the parallel surfaces oriented perpendicularly to the $c$ axis to avoid the superposition of the interference spectra of both ordinary and extraordinary refractive indexes. Furthermore, the pressure variations of the cell parameters " $a$ " and " $c$ " are such that the $\mathrm{c} / \mathrm{a}$ ratio remains practically constant up to 12 $\mathrm{GPa}^{6}{ }^{6}$ and both can be described by the same Murnaghan equation of state

$$
\frac{l(P)}{l_{0}}=\left(1+\frac{B_{0}^{\prime}}{B_{0}} P\right)^{-1 / 3 B_{0}^{\prime}},
$$

where $B_{0}=239 \mathrm{GPa}$ is the bulk modules and $B_{0}^{\prime}=0.9$ is its pressure derivative, both at $P=0 .{ }^{6}$

\section{EXPERIMENTAL RESULTS AND DISCUSSION}

The obtained experimental results for the relative change of the refractive index of sapphire up to $16 \mathrm{GPa}$ are presented in Fig. 2 together with the results of Davis and Vedam ${ }^{4}$ up to $0.7 \mathrm{GPa}$, showing a good agreement in the low-pressure range.

The significative change in the slope of $n(P)$ observed at pressures around $12 \mathrm{GPa}$ could be related to nonhydrostatic effects inside the DAC since the methanol-ethanolwater mixture solidifies between 12 and $14 \mathrm{GPa} .{ }^{9}$ To check this possibility, we performed an experiment using silicon oil as the pressure-transmitting medium and we found that the strong change in the slope of $n(P)$ occurred, in this

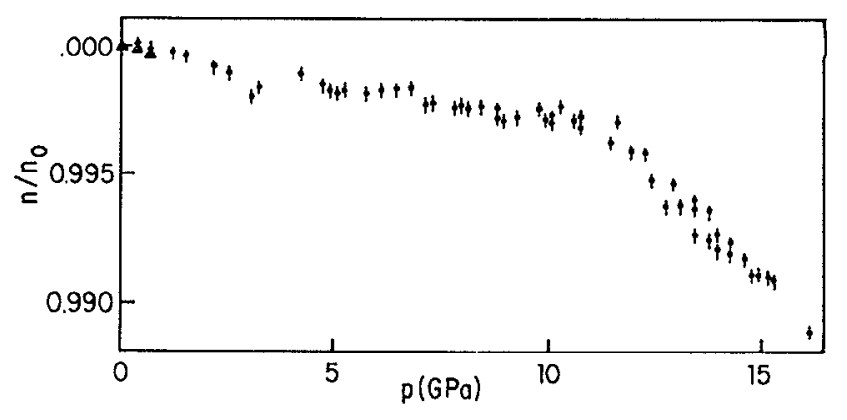

FIG. 2. Experimental results for the pressure dependence of the refractive index of sapphire up to $16 \mathrm{GPa}(\cdot)$, compared with the results of Davis and Vedam (see Ref. 4) $(\Delta)$. The experimental uncertainties for the pressure measurements are about $0.1 \mathrm{GPa}$ and $n / n o$ are represented by the vertical bars.

case, for pressures between 5 and $6 \mathrm{GPa}$, which corresponds to the solidification region of the oil used. ${ }^{9}$ Therefore, we can conclude that anisotropic stresses due to nonhydrostaticity inside the DAC should be responsible for this behavior. We then restricted our analysis to the region up to $12 \mathrm{GPa}$, where the pressure should be hydrostatic.

The classical Lorentz-Lorenz approach ${ }^{10}$ considers the ionic crystal as a collection of isolated entities, polarizable under the action of an electric field. In this context, the pressure variation of the refractive index $(n)$ is determined by two factors depending on $P$ : Density $(N)$ and electronic polarizability $(\alpha)$, according to the following relation

$$
\frac{n^{2}-1}{n^{2}+2}=\frac{4 \pi}{3} N \alpha \text {. }
$$

Although the factor $4 \pi / 3$ in Eq. (4) is strictly correct only for cubic lattices, we assume this relation to be valid also for sapphire ${ }^{11}$ because this crystal is isotropically compressed under hydrostatic pressures ${ }^{6}$ and we are interested only in relative changes in refractive index and polarizability.

Equation (4), together with the experimental results for $n(P)$ up to $12 \mathrm{GPa}$ and the Murnaghan equation of state for the density variation, provides the nearly linear volume dependence of $\alpha$ shown in Fig. 3. This behavior is

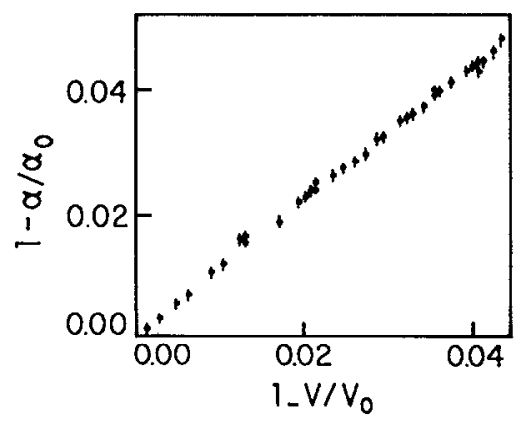

FIG. 3. Volume dependence of the polarizability for pressures up to 12 GPa. Vertical bars indicate the experimental uncertainties. 
in agreement with an early work of Mueller, ${ }^{12}$ which suggested that the decrease of $\alpha$ would be proportional to the volume decrease

$$
\frac{\Delta \alpha}{\alpha_{0}}=\Lambda_{0} \frac{\Delta V}{V_{0}},
$$

where $\Lambda_{0}$ is the strain polarizability constant, or Mueller parameter. The fit of the results of Fig. 3 provides $\Lambda_{0}$ $=1.128 \pm 0.017$, with a reduced $\chi^{2}$ of about 3.9 .

In the literature, there is an alternative definition of the Mueller parameter: ${ }^{10} \Lambda_{0}=(\delta \ln \alpha / \delta \ln V)_{T}$, that gives, after integration for a constant $\Lambda_{0}$,

$$
\frac{\alpha}{\alpha_{0}}=\left(\frac{V}{V_{0}}\right)^{\Lambda_{0}} .
$$

In this case, the fit of the results of Fig. 3 yields $\Lambda_{0}=1.130$ \pm 0.018 , with $\chi^{2}=3.85$, showing no significative improvement of the fitting.

Waxler and Weir ${ }^{5}$ pointed out qualitatively that the decrease of $\alpha$ would be accentuated at high pressures due to the intensification of the interatomic interactions. However, this was not observed in the present work up to 12 GPa. Moreover, a quadratic fitting of $\alpha(V)$ provides $\left(-\Delta \alpha / \alpha_{0}\right)=(1.21 \pm 0.07)\left(-\Delta V / V_{0}\right)-(3 \pm 2)(-\Delta V /$ $\left.V_{0}\right)^{2}\left(\chi^{2}=2.4\right)$, where the negative nonlinear coefficient, even with such a large uncertainty, indicates a small tendency of saturation of $\alpha$ with decreasing volume. It is important to point out that the equation of state and the compressibility data utilized play a significative role in the obtained behavior of $\alpha(V)$. For this reason we compared the results provided by two sets of distinct values of $B_{0}$ and $B_{0}^{\prime}$ found in the literature ${ }^{6}$ and both indicate the small tendency of saturation of $\alpha(V)$.

According to the shell model, ${ }^{13}$ the ion is considered to be composed of a spherical shell of outermost electrons, and a core consisting of the nucleus and the inner electrons. These two parts are held together by a spring constant $k_{i}$, and the electronic polarizability is given by $\alpha=(Z e)^{2} / k_{i}$, where $-Z e$ is the electronic charge of the shell. Therefore, we can write $\Lambda_{0}=-d \ln k_{i} / d \ln V$, showing that $\Lambda_{0}$ is a kind of "Grüneisen parameter" for this dynamic system.

In fact, in the case of the harmonic oscillator model for the lattice vibrations, ${ }^{14}$ the Grüneisen parameter is defined as $\gamma=-d \ln \omega / d \ln V$, where $\omega$ is the frequency of the ionic oscillatory motion, proportional to the square root of the lattice spring constant $k_{l}$, so it is possible to write $\gamma=(-1 / 2) d \ln k_{l} / d \ln V$. It is interesting to point out that, in the case of sapphire, $\gamma$ is practically independent of pressure $^{15}$ and we obtained a constant value also for $\Lambda_{0}$, indicating that, despite of the lattice vibrations and the electronic polarizability are different, but coupled, dynamical systems, the behaviors of the lattice and ionic spring constants seem to be similar for small volume variations.

We also used the results of $\alpha(V)$ to test some simple semiempirical relations found in the literature, relating the anion polarizability $\alpha^{-}$to the interionic distance $r$. In the case of ionic crystals, the anion optical behavior is generally dominant, since it has usually a bigger size than the
TABLE I. (a) Semiempirical models for $\alpha^{-}(V)$, and (b) results for the free parameters, where $C$ is an adjustable parameter, $\alpha_{f}^{-}$is the free-anion

\begin{tabular}{|c|c|c|}
\hline (a) Model & \multicolumn{2}{|c|}{ Relation } \\
\hline $\begin{array}{l}1 \text { W.C. } \\
2 \text { Coker }^{b} \\
3 \text { Coker }^{b} \\
4 \text { Coker }^{b} \\
5 \text { Coker }^{b}\end{array}$ & \multicolumn{2}{|c|}{$\begin{array}{c}\alpha^{-} / \alpha_{f}^{-}=\exp \left(-C / r^{2}\right) \\
\alpha^{-} / \alpha_{f}^{-}=\left(1+C r^{-3}\right)^{-1} \\
\alpha^{-} / \alpha_{f}^{-}=\left(1+C r^{-4}\right)^{-1} \\
\alpha^{-} / \alpha_{f}^{-}=\left\{1-\exp (-C r)\left[1+C r+(C r)^{2} / 2 !\right]\right\} \\
\alpha^{-} / \alpha_{f}^{-}=\left\{1-\exp (-C r)\left[1+C r+(C r)^{2} / 2 !+(C r)^{3} / 3 !\right]\right\}\end{array}$} \\
\hline (b) & Parameter & $\alpha_{f}^{-}\left(10^{-3} \mathrm{~nm}^{3}\right)$ \\
\hline $1 C / r_{0}^{2}=$ & $1.68 \pm 0.02$ & $22.7 \pm 0.5$ \\
\hline $2 C / r_{0}^{3}=$ & $-8.5 \pm 1$ & $-31 \pm 4$ \\
\hline $3 C / r_{0}^{4}=$ & $5.5 \pm 0.5$ & $27 \pm 2$ \\
\hline $4 C r_{0}=$ & $-0.51 \pm 0.06$ & $-127 \pm 50$ \\
\hline $5 C r_{0}=$ & $0.79 \pm 0.07$ & $477 \pm 130$ \\
\hline
\end{tabular}
polarizability, and $r$ is the nearest neighbor distance.

asee Ref. 18.

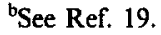

cation and it is strongly affected by the crystal environment. ${ }^{16}$ For sapphire $\left(\mathrm{Al}_{2} \mathrm{O}_{3}\right)$, we consider that $\alpha=2 \alpha^{+}$ $+3 \alpha^{-}$, where $\alpha^{+}=0.052 \times 10^{-3} \mathrm{~nm}^{317}$ is the cation polarizability, assumed to be independent of pressure. The relations for $\alpha^{-}(r)$ are presented in Table I together with the values of the adjustable parameter $C$ and of the freeanion polarizability $\alpha_{f}^{-}$, yielded by the fit of the results derived from the data of Fig. 3. Despite the fact that all of these relations provide a reasonable value for $\alpha_{0}^{-}$around $4 \times 10^{-3} \mathrm{~nm}^{3}$, most of them yields negative or values too large for the free-anion polarizability.

\section{CONCLUSIONS}

In this work we presented experimental results for the pressure dependence of the refractive index of sapphire up to $16 \mathrm{GPa}$, which, in the low pressure range, are in good agreement with the previous results of Davis and Vedam up to $0.7 \mathrm{GPa}$. For pressures above $12 \mathrm{GPa}$, anisotropic stresses inside the DAC induce a strong deviation in the behavior of $n(P)$.

The volume dependence of the electronic polarizability, obtained by the Lorentz-Lorenz relation, is nearly linear in the hydrostatic range of pressure, well described by a constant Mueller parameter.

According to the shell model, a constant value for $\Lambda_{0}$ implies in a simple scaling factor for the volume dependenc of the ionic spring constant, representing the interaction between shell and core.

The semi-empirical models found in the literature, relating the anion polarizability and the interionic distance, seem to be not very adequate to describe the obtained results.

\section{ACKNOWLEDGMENTS}

We are grateful to Dr. B. Blanzat and Dr. J. M. Leger from the Laboratoire de Physico-Chimie des Materiaux, CNRS, Meudon, France, for the helpful contributions in 
the experimental technique and discussions. This work was supported in part by CAPES, CNPq, FAPERGS, and FINEP.

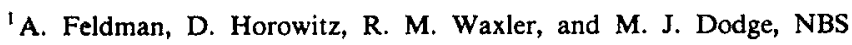
Technical Note 993 (1978).

${ }^{2}$ N. M. Balzaretti and J. A. H. da Jornada, High Pressure Res. 2, 183 (1990).

${ }^{3}$ k. Vedam and E. D. D. Schmidt, Solid State Commun. 3, 373 (1965).

${ }^{4}$ T. A. Davis and K. Vedam, J. Appl. Phys. 38, 4555 (1967).

${ }^{5}$ R. M. Waxler and C. E. Weir, J. Res. Nat. Bur. Stand. A 69A, 325 (1965),

'Y. Sato and S. Akimoto, J. Appl. Phys. 50, 5285 (1979).

${ }^{7}$ N. M. Balzaretti, J. T. N. Medeiros, and J. A. H. da Jornada, Science and Technology of New Diamond, edited by S. Sato, O. Fukunaga, and M. Yoshikawa (KTK, 1990), pp. 441-444.

${ }^{8}$ S. S. Ballard, K. A. McCarthy, and W. L. Wolfe, Optical Materials for
Infrared Instrumentation (University of Michigan, Michigan, 1959), p. 24.

${ }^{9}$ J. M. Leger, A. M. Redon, and C. Chateau, Phys. Chem. Minerals 17, 161 (1990).

${ }^{10}$ H. Coker, J. Phys. Chem. Solids 40, 1079 (1979).

${ }^{11}$ K. Vedam and T. A. Davis, J. Opt. Soc. Am. 57, 1140 (1967).

${ }^{12}$ H. Mueller, Phys. Rev. 47, 947 (1935).

${ }^{13}$ L. K. Banerjee and A. N. Basu, Phys. Rev. B 20, 1706 (1979).

${ }^{14} \mathrm{M}$. Born and K. Huang, Dynamical Theory of Crystal Lattices ( Clarendon, Oxford, 1968).

${ }^{15}$ G. H. Watson, Jr., W. B. Daniels, and C. S. Wang, J. Appl. Phys. 52, 956 (1981).

${ }^{16}$ P. N. Fowler, R. W. Munn, and P. Toole, Chem. Phys. Lett. 176, 439 (1991).

${ }^{17}$ L. Pauling, Proc. R. Soc. A 114, 181 (1927).

${ }^{18}$ J. N. Wilson and R. M. Curtis, J. Phys. Chem. 74, 187 (1970).

${ }^{19}$ H. Coker, J. Phys. Chem. 80, 2078 (1976). 